\title{
Projektkooperationen und Wissensaustausch zur Förderung der Patientensicherheit
}

Paula Bezzola, Marc-Anton Hochreutener

Korrespondenz:

Paula Bezzola, MPH

Dr. med. Marc-Anton Hochreutener Stiftung für Patientensicherheit

Asylstrasse 41

CH-8032 Zürich

Tel. 0432437670

Fax 0432437671

info@patientensicherheit.ch

www.patientensicherheit.ch
Kooperationen und organisierter Wissensaustausch helfen $\mathrm{zu}$ verhindern, dass immer wieder dieselben Fehler passieren. Die Stiftung für Patientensicherheit wurde 2003 gegründet. Als Netzwerkorganisation arbeitet sie in zahlreichen Projekten mit verschiedenen Partnern zusammen, um die Patientensicherheit zu fördern. Unter einem breiten Patronat organisiert sie beispielsweise zusammen mit der Schweizerischen Gesellschaft für Qualitätsmanagement im Gesundheitswesen SQMH am 13. und 14. September 2007 einen Kongress in Bern zum Thema «Patientensicherheit Schweiz: Aktivitäten - Stolpersteine - Perspektiven». An dem Anlass werden richtungweisende Projekte vorgestellt und der interdisziplinäre Austausch gefördert.

\section{Sicherheitsprobleme erkennen und analysieren}

Aus Fehlern lernt man - diese Binsenwahrheit rührt im Gesundheitswesen an ein Dogma, nämlich die Unfehlbarkeit der Medizin. Selbstverständlich treten auch im Gesundheitswesen Fehler auf. Doch nur bekannte und dokumentierte Fehler lassen sich ein zweites Mal vermeiden - entsprechend einem weiteren Bonmot, wonach man zwar einen Fehler machen darf, aber nicht zweimal den gleichen. Der erste Schritt im Kreislauf des klinischen Risikomanagements zur Verbesserung der Patientensicherheit ist deshalb, Sicherheitsprobleme im System zu erkennen und sich einzugestehen (Abb. 1).

\section{Kooperationen eingehen}

Die Stiftung für Patientensicherheit sucht gezielt Kooperationen und die Zusammenarbeit in Netzwerken. Denn die Dokumentation von Fehlern und das Erkennen der relevanten Sicherheitsprobleme lässt sich alleine kaum bewältigen: Wissen über klinisches Risikomanagement, Erfahrungen mit Fehlerkultur bzw. Sicherheitskultur sowie die Handlungsbereitschaft der Akteure im Gesundheitswesen müssen zusammenfliessen. Zusammenarbeit und geteiltes Wissen bilden die Grundlage dafür, Lösungen zur Fehlervermeidung zu entwickeln, Sicherheitsprobleme im System zu mindern und die Sicherheitskultur zu fördern.
So besteht beispielsweise eine erfolgreiche Zusammenarbeit in mehreren Projekten zwischen der Stiftung für Patientensicherheit und der Schweizerischen Gesellschaft für Anästhesiologie und Reanimation (SGAR). Im Rahmen einer weiteren, internationalen Kooperation mit Experten aus England, Charles Vincent und Sally Adams, wird die Methodik der systemischen Analyse gemäss London Protocol in der Schweiz verbreitet [1]: Das London Protocol zeichnet sich durch eine klare Logik der Fehleranalyse aus, die auf das System und die verschiedenen beeinflussenden Faktoren fokussiert. Ziel ist, dass die Organisation für die Zukunft lernen kann und so organisiert wird, dass Fehler systematisch vermieden werden.

\section{Von der Kooperation zur Wissensverbreitung}

Der Kreislauf zur Fehlervermeidung und Förderung der Sicherheit schliesst sich, wenn die Erkenntnisse und Verbesserungsmethoden auch verbreitet werden: in Spitälern, Arztpraxen und weiteren Institutionen des Gesundheitswesens. So hat die Stiftung für Patientensicherheit zusammen mit der SGAR Empfehlungen zur Kommunikation mit Patienten und Angehörigen nach einem Behandlungszwischenfall publiziert $[2,3]$. Im Rahmen des Projektes CIRRNET (Critical Incident Reporting and Reacting Network) beteiligen sich 24 Spitäler an einem Pilotprojekt, bei dem die kritischen Zwischenfälle in einem gemeinsamen Datenpool erfasst und bearbeitet werden. Das Wissen aus diesem Pool kann wiederum verbreitet werden. In Kooperation mit der fmch werden in nächster Zeit Empfehlungen zur Vermeidung von Eingriffsverwechslungen herausgegeben.

Aus- und Weiterbildung sind die Grundlagen der Wissensverbreitung. Dazu hat die Schweizerische Akademie der Medizinischen Wissenschaften (SAMW) Empfehlungen für die Integration des Themas Patientensicherheit in der Aus- und Weiterbildung erarbeitet, an denen die Stiftung intensiv mitgearbeitet hat. Kurse zur systemischen Analyse von Behandlungszwischenfällen nach dem London Protocol werden die Methodik in der Schweiz verbreiten helfen und eine gemeinsame Sprache in diesem Gebiet 
Abbildung 1

Auftrag der Stiftung für Patientensicherheit.

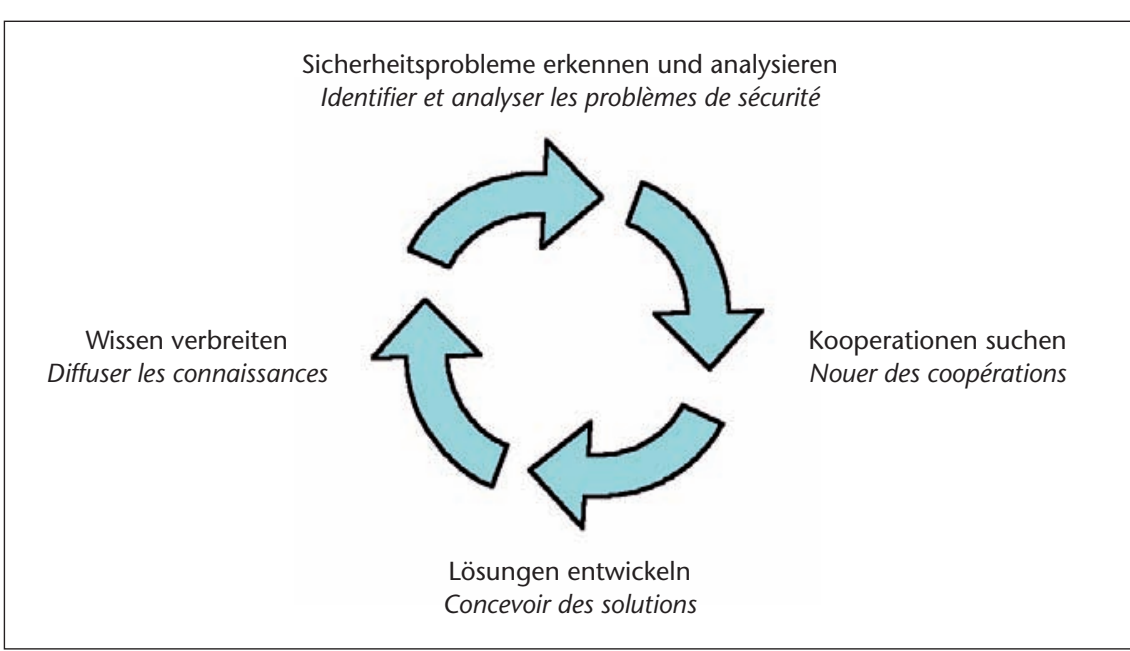

ermöglichen. Auch die FMH unterstützt ein Projekt, bei dem ermittelt wird, wie bei Zwischenfällen betriebsintern vorzugehen ist. Dabei wird ein Handlungsrahmen für den systematischen und konstruktiven betriebsinternen Umgang mit Zwischenfällen entwickelt. Dies dient nicht nur der Patientensicherheit, sondern trägt auch dazu bei, Phänomene wie Burn-out und Berufsausstiege aufgrund der Verunsicherung durch Fehler aufzufangen. Das Projekt wird in Kooperation mit dem Institut für Sucht- und Gesundheitsforschung durchgeführt.

\section{Kongress «Patientensicherheit Schweiz: Aktivitäten - Stolpersteine - Perspektiven" in Bern,}

13. und 14. September 2007

Die Stiftung für Patientensicherheit Schweiz und die Schweizerische Gesellschaft für Qualitätsmanagement im Gesundheitswesen (SQMH) organisieren gemeinsam unter einem breiten $\mathrm{Pa}$ tronat die Tagung «Patientensicherheit Schweiz: Aktivitäten - Stolpersteine - Perspektiven». Sie bietet eine breite Auslegeordnung der Aktivitä- ten zur Vermeidung von Fehlern in der Medizin. Zusammen mit internationalen Pionieren (z.B. Lucian Leape aus den USA [4] und Charles Vincent aus England [5]) und nationalen Opinionleaders und Experten werden Perspektiven entwickelt und die Netzwerkbildung sowie der Wissensaustausch gefördert. Der erste Tag des Kongresses ist den Themen Kultur und Kommunikation gewidmet. Am zweiten Tag werden ausgesuchte Handlungsfelder wie Ausbildung oder Medikationssicherheit genauer beleuchtet. In drei Blocks mit je 6 Parallelveranstaltungen werden zudem zahlreiche konkrete Ansätze zur Verbesserung der Patientensicherheit vorgestellt. Etliche interaktive Workshops ermöglichen, sich vertieft mit spezifischen Themen und Methoden auseinanderzusetzen.

\section{Informationen zur Tagung}

«Patientensicherheit Schweiz:

Aktivitäten - Stolpersteine - Perspektiven» in Bern, 13. und 14. September 2007, und zu weiteren Leistungen und Kooperationspartnern der Stiftung für Patientensicherheit: www.patientensicherheit.ch und Website des Projektes CIRRNET: www.cirrnet.ch

\section{Literatur}

1 Taylor-Adams S, Vincent C. Systems analysis of clinical incidents: the London protocol. Clinical Risk. London, Clinical Safety Research Unit, Imperial College London, Department of Surgical Oncology and Technology. 2004;10:211-20.

2 Harvard Hospitals. When things go wrong responding to adverse events. 2006.

3 Stiftung für Patientensicherheit. Wenn etwas schief geht - Kommunizieren und Handeln nach einem Zwischenfall. schriftenreihe patientensicherheitschweiz. Nr. 1. Zürich: Stiftung für Patientensicherheit; 2006.

4 Leape LL. Error in medicine. JAMA. 1994; 272:1851-7.

5 Vincent C. Patient Safety. London: Churchill Livingstone; 2006. 